\title{
TECHNIQUE OF CREATING NEW CONSTRUCTIONS OF ACOUSTIC AND MAGNETIC DEVICES BASED ON THEORY OF SIMILARITY AS DEVELOPMENT OF THEORY OF EXPERIMENT
}

\author{
Alexey Korzhakov ${ }^{1}$, Sergei Oskin ${ }^{2}$, Svetlana Korzhakova ${ }^{1}$ \\ ${ }^{1}$ Adyghe State University, Russia; ${ }^{2}$ Kuban State Agrarian University, Russia \\ korhakov-av@yandex.ru,mechanization@kubsau.ru ,
}

\begin{abstract}
The article considers the theory of similarity as a development of the theory of experiment. Summarizing the experimental data obtained in the study of the acoustic and magnetic device designed for nonreagent treatment of liquid, the task of transferring to an unlimitedly large class of devices, which, in accordance with the criteria obtained, is recognized as similar to this device, is set. The work establishes the criteria connecting the geometric parameters of different types of devices and criteria that establish the ratio of the environmental temperature to the thermal regime of the device. The similarity of heat transfer conditions on the surface of the device is provided, and the relation between the scale of the temperature and the power, that is lost when the ferrite and the windings of the device are heated, is determined. The criterion of the heat transfer process is established. In accordance with the task, on the basis of the third theorem of similarity, a group of criteria is chosen that ensures similarity of the model to the proposed original on selected indicators.
\end{abstract}

Keywords: theory of similarity, experimental statistical methods, regression model, plans for experiments.

\section{Introduction}

Improvement of the effectiveness of the experiment is due to correct conducting of experimental studies and their processing. The existing methods of conducting experiments and processing their results do not reflect the current state of affairs. A quick change of the instrument base, development of software containing modules with statistical processing of data, computer modeling further and further leads researchers away from physical modeling, which ultimately interferes with production of real objects. Creation of physical models in large-scale variants becomes possible on the basis of the theory of similarity. The designated problem is solved by creating a new technique that allows creating large-scale physical models with determination of their parameters level.

\section{Materials and methods}

Agricultural technology of growing vegetables in greenhouses requires accurate maintenance of the temperature regime. Geothermal sources are used for heat supply of greenhouse complexes. Heated water from the greenhouse is supplied to the heat exchange unit. Geothermal heat double loops contour with intermediate heat exchangers that are used to prevent scale formation of hydroponic greenhouses equipment and communications. Geothermal water heats fresh soft water, which is supplied further for consumer needs. The heat exchanger and the communication of the primary circuit of the system are corroded because they contact with the geothermal water (heat carrier). It is known that the thermal characteristics deteriorate; the hydraulic resistance of pipelines increases, their complete blockage and system failure are possible in the process of scaling. The process of cleaning the heat exchanger continues for at least two weeks and technical regulation depends on the aggressiveness of geothermal water. It is technologically impossible to maintain the temperature in greenhouses during technical regulations, so, if the regulations coincide with the low ambient temperature, harvest will be lost partially or completely.

Reagent (chemical) geothermal water treatment methods do not allow to prevent geothermal water heating system scale formation, and work upon scale formation. The conducted research of nonchemical water treatment leads to the conclusion that a significant scaling reduction will be achieved with the acoustic-magnetic treatment of geothermal water. The highest efficiency of water treatment is achieved by combining ultrasound with a magnetic rotating field. This method has received patents of the Russian Federation [1-4].

Observations of calcium carbonate deposition processes in geothermal water with various chemical composition, mineralization, temperature and pressure at the wellhead, suspended particles resume that calcium carbonate scale formation locates along the route of water transportation. Various water pressure and temperature values determine scale locations, all other things being equal. Most 
often, calcium carbonate scale formations locate behind the valves, at places of a sharp pressure dropping and pipe knees.

Thereby, it is necessary to create energy-saving modification of acoustic-magnetic devices used at geothermal heat sources for geothermal water treatment. The designs of acoustic-magnetic devices are determined with prototypes. It is proposed to optimize the acoustic-magnetic devices by usage of experimental statistical methods with simultaneous application of the theory of similarity.

The general scheme for planning experiments for solving extremal problems consists of the following stages: statement of the problem, selection of optimization parameters, selection of factors, linear planning and construction of a linear model, search for an extremum area, description of the extremum area and interpretation of the results [5].

Having set the task of identifying the factors that affect the characteristics of the acoustic magnetic device, we form the goal of transferring the results obtained on the model to the original, as well as finding ways to change the characteristics of the device in the desired direction.

There are many different conditional indicators and empirical coefficients for calculating the parameters of magnetic devices. But all of them have one essential drawback: a narrow range of parameters of the construction that work under certain conditions. The criteria of similarity have some advantage that does not depend on the size of the structures being compared, as they are dimensionless complexes.

Composition of the criteria of similarity shows which values should be measured in experience so that their results should be comparable. The obtained experimental data, recorded in the criterial form, can be used to generalize the results of experiments in order to create new constructions or to compare with the existing constructions. To create acoustic magnetic devices of a new design, it is necessary to consider geometric, electromagnetic, thermal, and mechanical similarities.

The flow of processes in the acoustic magnetic field is characterized by the following criteria of similarity:

- generalized criteria for geometric parameters of various types and designs of an acoustic magnetic device;

- criteria connecting the physical parameters, the parameters of the environment and the acoustic magnetic device;

- criterion establishing the relation of the temperature of the environment to the thermal mode of operation of the device;

- criteria establishing the initial state of the circuit containing mutual inductances;

- criterion that establishes conditions on the boundaries of the system and the cavitating fluid.

This article will focus on a brief description of the criteria, connecting the geometrical parameters of different types of devices and criteria for establishing the ratio of ambient temperature to the heat mode of the device.

Let us consider the generalized criteria for the geometric parameters of the construction of an acoustic magnetic device.

The core of the acoustic magnetic device is completely characterized by three linear dimensions: $a=R_{H}-R_{B} ; b$ is the width of the magnetic circuit: $c=D_{i n}=d$.

Let us take the size a as the basic one. Using a system of relative units, one can determine all other dimensions and geometric parameters, depending on this basic size and two dimensionless parameters:

$$
\pi_{1}=\frac{c}{a}, \pi_{2}=\frac{b}{a},
$$

where $\pi_{1}$ - the ratio of the inner diameter to the base dimension;

$\pi_{2}$ - the ratio of the width of the ring to the base dimension.

For the acoustic magnetic device, the value of the coefficient of the fill of the window with winding $\pi_{3}$ is found from the expression: 


$$
\pi_{3}=\frac{\pi_{1}-\frac{d_{0}}{a}}{\pi_{1}},
$$

where $d_{0}$ - the size of the hole for passage of the shuttle.

In order to find the dimensions of the device, first of all it is necessary to determine the constants $\pi_{1}, \pi_{2}, \pi_{3}, \pi_{4}$, calculate the size of its basis size $a$ considering $\pi_{4}$ as the ratio of the losses in the core to the losses in the winding:

$$
\pi_{4}=\frac{P_{c}}{r_{0} i^{2}} .
$$

As a result, we obtain a criterion equation of geometric parameters of different types and designs of the acoustic magnetic device:

$$
\pi_{5}=f_{1}\left(\pi_{1}, \pi_{2}, \pi_{3}, \pi_{4}\right) .
$$

The steady-state value of the superheat temperature of the acoustic magnetic device depends on the efficiency of the heat sink from its surface. The efficiency is determined by the $\alpha$ coefficient of heat transfer, which characterizes the power allocated to the unit of the surface with a temperature change of one degree.

The heat exchange of the toroidal acoustic magnetic device with the environment is realized through two side and two end surfaces. Since it is not known beforehand which factors influence the heat transfer coefficient values and how they do it under the specific operation conditions of the acoustic magnetic device, we assume that the cooling conditions of the acoustic magnetic device are the same over the entire surface, and all the heat exchange coefficients are equal:

$$
\alpha_{1}=\alpha_{2}=\alpha_{3}=\alpha_{4}=\alpha_{0} .
$$

You can apply the expression [6] to calculate the parameters. The steady superheat temperature of the toroidal transformer:

$$
\theta-T_{0}=\frac{r_{0} i^{2}+P_{c}}{\alpha_{0} F_{0}-\chi r_{0} i^{2}},
$$

where $r_{0}$ - the impedance of the device;

$\chi$ - the temperature coefficient of resistance;

$i$ - the total value of the device current;

$\theta$ - the average temperature in the volume of the device;

$T_{0}$ - the ambient temperature;

$F_{0}$ - the surface area of the device;

$r_{0} i^{2}+P_{c}$ - the total power of losses;

$P_{c}$ - the loss in the magnetic circuit.

The power lost due to eddy currents $P_{v}$, can be calculated by the formula:

$$
P_{v}=\frac{\delta_{1}}{\rho}\left(b \cdot s(t) \cdot B_{M} \cdot 10^{-5}\right)^{2},
$$

where $\delta_{1}$ - the coefficient depending on the magnetostrictive material;

$\rho$ - the density of the magnetostrictive material;

$b$ - the thickness of the ring;

$s=s(t)$ is the frequency of the current applied to the device;

$B_{M}$ - the amplitude value of the magnetic induction (found along the curves of magnetostriction).

The power loss due to the hysteresis $P_{g}$ is determined by the expression: 


$$
P_{g}=\frac{\delta_{2}}{\rho} s(t) \cdot 10^{-2}\left(\frac{B_{M}}{1000}\right)^{1.6},
$$

where $\delta_{2}$ - a coefficient depending on the magnetostrictive material.

The losses in the magnetic circuit consist of losses for eddy currents and hysteresis [7]

$$
P_{c}=P_{v}+P_{g} \text {. }
$$

Dependence (6) is realized, if the denominator of the fraction is positive. This expression includes the surface area of the device. Suppose that the value of the area of the device and the magnetic circuit are constant and do not depend on the power of the device.

Let us introduce the coefficient of similarity, as the ratio of the area of the device and the magnetic circuit:

$$
\pi_{6}=\frac{F_{0}}{F},
$$

where $F=2 \pi\left(R_{H}+R_{B}\right)\left(b+R_{H}-R_{B}\right)$ - the surface area of the magnetic circuit, where $R_{H}$ is the outer radius of the magnetic circuit;

$R_{B}$ - the internal radius;

$b$ - the height of the magnetic circuit;

$F_{0}=2 \pi\left(R_{\text {out }}^{2}-R_{\text {in }}^{2}\right)+2 \pi\left(R_{\text {out }}+R_{\text {in }}\right)$ - the surface area of the device through which heat exchange with the environment takes place, where $R_{\text {in }}$ is the internal radius of the device window;

$R_{\text {out }}$ - the outer radius of the device;

$h$ - the height of the device.

Let us substitute the value $F_{0}$ from (10) into expression (6):

$$
\theta-T_{0}=\frac{r_{0} i^{2}+P_{c}}{\pi_{6} \alpha_{0} F_{0}-\chi r_{0} i^{2}} .
$$

We introduce the generalized coefficient of heat exchange similarity $\pi_{7}=\pi_{6} \alpha_{0}$, then the steadystate overheat temperature of the acoustic magnetic device can be written down as:

$$
\theta-T_{0}=\frac{r_{0} i^{2}+P_{c}}{\pi_{7} F-\chi r_{0} i^{2}} .
$$

The total value of the device current consists of the active and reactive components [8]:

$$
i=\sqrt{\left(\frac{m_{c} P_{0}}{E}\right)^{2}+\left(\frac{E l}{2 \pi s(t) \mu_{0} \mu S w^{2}}\right)^{2}},
$$

where $E-$ the EMF of the windings of the device;

$m_{c}-$ the mass of the device;

$S$ - the cross-sectional area of the magnetic circuit;

$\mu_{0}$ - the magnetic constant equal to $4 \pi \cdot 10^{-7} \mathrm{H} \cdot \mathrm{m}^{-1}$;

$\mu$ - the relative magnetic permeability of the magnetic circuit material;

$w$ - the number of turns of the device winding;

$P_{0}$ - the specific power of losses in ferrite;

$s=s(t)-$ the frequency of the current.

The weight of the emitter $m_{c}$ is determined by the expression:

$$
m_{c}=\frac{\pi \rho_{c} h k_{c}}{4}\left(\left(2 R_{\text {out }}\right)^{2}-\left(2 R_{\text {in }}\right)^{2}\right),
$$

where $\rho_{c}$ - the density of ferrite. 
The value of $P_{0}$ can be determined from the formula:

$$
P_{c}=m_{c} P_{0} .
$$

When calculating the parameters of the device at high frequencies because of the manifestation of the surface effect, the active resistance of the device can be determined by the formula:

$$
r_{a}=\rho \frac{w_{k} l_{0} n_{k} k_{r}}{q}
$$

where $w_{k}$ is the number of turns of one coil;

$l_{0}$ - an average length of a winding turn;

$n_{k}$ - the number of coils in the device;

$k_{r}$ - the coefficient of resistance increase, the value of which belongs to the interval $[1.05 ; 1.3]$;

$\rho$ - the electrical resistance of the conductor;

$q$ - the cross-section of the conductor.

In order to test the hypothesis (12), a series of experiments was carried out with devices of different power. The analysis of the obtained results allows calculating this coefficient of similarity. Several percent made up difference for devices of different power, i.e. in fact, $\pi_{6}=$ const $=5.25$. The criterion $\pi_{7}$ is an empirical value, the numerical value of which was obtained in the process of comparing the results of testing the device with theoretical data.

To verify the theoretical data, we conducted the thermal tests of an acoustic magnetic device. Devices were tested in full load operation mode. Besides, it is necessary to ensure the similarity of heat transfer conditions on the surface of the device and to establish a relationship between the scale of temperature and the power lost on heating the ferrite and the windings of the device. The similarity of the heat transfer process is established by the criterion:

$$
\pi_{8}=\frac{\left(\theta-T_{0}\right)\left(\pi_{7} F-\chi r_{0} i^{2}\right)}{r_{0} i^{2}+P_{c}} .
$$

Let us consider the application of the experimental statistical methods with simultaneous application of the theory of similarity for studying the processes of acoustic magnetic effects on scale [5].

The processes taking place in the acoustic magnetic device can be represented by the dependence

$$
\begin{gathered}
f\left(P_{1}, P_{2}, \ldots, P_{k}, \ldots, P_{S}, \ldots, P_{m}\right)=0 \\
P_{1}=b ; P_{2}=a_{1} ; P_{3}=d_{0} ; P_{4}=d_{0} ; P_{5}=i ; P_{6}=P_{c} ; P_{7}=h ; P_{8}=P_{\text {out }} ; P_{9}=\Theta ; P_{10}=r_{0} .
\end{gathered}
$$

The characteristic of the process connecting the anti-scale indicator with the ongoing processes in the acoustic magnetic field is represented by the established criteria of similarity:

$$
\pi_{1}^{\prime}=f_{1}\left(\pi_{1}^{\prime}, \pi_{2}^{\prime}, \pi_{3}^{\prime}, \pi_{4}^{\prime}, \pi_{5}^{\prime}\right),
$$

where $\pi_{2}, \pi_{3}, \pi_{4}, \pi_{6}, \pi_{8}$ are equal accordingly $\pi_{1}^{\prime}, \pi_{2}^{\prime}, \pi_{3}^{\prime}, \pi_{4}^{\prime}, \pi_{5}^{\prime}$.

The quantity $\pi_{1}^{\prime}$ is the objective function, in the search for which the theory of similarity allows to reduce the number of variables from $m$ dimensional quantities to $m-k$ dimensionless. Applying methods of factor planning of the experiment, we obtain a criteria equation of regression of the form

$$
\pi_{1}^{\prime}=f_{1}\left(\pi_{2}^{\prime}, \pi_{3}^{\prime}, \ldots, \pi_{m-k}^{\prime}\right)=b_{0}+\sum_{i=2}^{m-k} b_{i} \pi_{i}+\sum_{\substack{i \neq j \\ j=2}}^{m-k} b_{i j} \pi_{i} \pi_{j}+\sum_{\substack{i \neq j \\ j=2}}^{m-k} b_{i i} \pi_{i}^{2}+\ldots
$$

where $m$ - the total number of parameters studied;

$k-$ the number of independent parameters.

The peculiarity of the application of this method is that the researcher deals not with individual factors, as usual, but with generalized ones. 
The application of the classical approach to the study of criterial ratios is somewhat complicated, since it is impossible to specify the step of variation by the criteria of similarity, regardless of the step of varying the factors included in these criteria.

In this case, two approaches can be used: 1) to repeatedly simulate the factors of the process at random within a given variation range and, for each random implementation, calculate the values of the criteria of similarity, then determine the step of varying the similarity criteria; 2 ) to set the step of varying the factors of the process and on this basis determine the value of the step of varying the criteria of similarity [9].

Further, according to the methods of experiment planning, the necessary experiment plan is selected and the experiment planning matrix (Table 1) is compiled for one of the series of the experiments.

This matrix differs from the usual one as it indicates not only the values of the criteria of similarity, but also the value of the parameters corresponding to a given numerical value of the criteria of similarity. In this case, the step of deviation of the criteria of similarity is determined by the variation of the parameters included in them [9].

Effective and factor characteristics for a linear model

Table 1

\begin{tabular}{|c|c|c|c|c|c|c|c|c|c|c|c|c|c|}
\hline \multirow{2}{*}{$Y$} & \multirow{2}{*}{$P_{0}$} & \multicolumn{2}{|c|}{$\pi_{1}^{\prime}$} & \multicolumn{2}{|c|}{$\pi_{2}^{\prime}$} & \multicolumn{2}{|c|}{$\pi_{3}^{\prime}$} & \multicolumn{2}{|c|}{$\pi_{4}^{\prime}$} & \multicolumn{2}{|c|}{$\pi_{5}^{\prime}$} & \multicolumn{2}{|c|}{$\pi_{k}^{\prime}$} \\
\hline & & $P_{11}$ & $P_{12}$ & $P_{21}$ & $P_{22}$ & $P_{31}$ & $P_{32}$ & $P_{41}$ & $P_{42}$ & $P_{51}$ & $P_{52}$ & $P_{k 1}$ & $P_{k 2}$ \\
\hline \multirow{2}{*}{$Y_{1}$} & \multirow{2}{*}{$\pi_{01}^{\prime}(1)$} & \multicolumn{2}{|c|}{$\pi_{11}^{\prime}(-)=0.75$} & \multicolumn{2}{|c|}{$\pi_{21}^{\prime}(-)=0.5$} & \multicolumn{2}{|c|}{$\pi_{31}^{\prime}(-)=0.00034$} & \multicolumn{2}{|c|}{$\pi_{41}^{\prime}(-)=5.01$} & \multicolumn{2}{|c|}{$\pi_{51}^{\prime}(-)=0.01$} & \multicolumn{2}{|c|}{$\pi_{k 1}^{\prime}$} \\
\hline & & (-) $P_{11}$ & (-) $P_{12}$ & (-) $P_{21}$ & (-) $P_{22}$ & $(-) P_{31}$ & (-) $P_{32}$ & (-) $P_{41}$ & (-) $P_{42}$ & $(-) P_{51}$ & (-) $P_{52}$ & $P_{k 1}$ & $P_{k 2}$ \\
\hline \multirow{2}{*}{$Y_{2}$} & \multirow{2}{*}{$\pi_{02}^{\prime}(1)$} & \multicolumn{2}{|c|}{$\pi_{12}^{\prime}(+)=0.46$} & \multicolumn{2}{|c|}{$\pi_{22}^{\prime}(+)=0.38$} & \multicolumn{2}{|c|}{$\pi_{32}^{\prime}(-)=0.00034$} & \multicolumn{2}{|c|}{$\pi_{42}^{\prime}(-)=5.01$} & \multicolumn{2}{|c|}{$\pi_{52}^{\prime}(+)=0.13$} & \multicolumn{2}{|c|}{$\pi_{k 2}^{\prime}$} \\
\hline & & $(+) P_{11}$ & $(+) P_{12}$ & $(+) P_{21}$ & $(+) P_{22}$ & $(-) P_{31}$ & $(-) P_{32}$ & $(-) P_{41}$ & $(-) P_{42}$ & $(+) P_{51}$ & $(+) P_{52}$ & $P_{k 1}$ & $P_{k 2}$ \\
\hline \multirow{2}{*}{$Y_{3}$} & \multirow{2}{*}{$\pi_{03}^{\prime}(1)$} & \multicolumn{2}{|c|}{$\pi_{13}^{\prime}(+)=0.46$} & \multicolumn{2}{|c|}{$\pi_{23}^{\prime}(-)=0.5$} & \multicolumn{2}{|c|}{$\pi_{33}^{\prime}(+)=0.00063$} & \multicolumn{2}{|c|}{$\pi_{43}^{\prime}(-)=5.01$} & \multicolumn{2}{|c|}{$\pi_{53}^{\prime}(+)=0.13$} & \multicolumn{2}{|c|}{$\pi_{k 3}^{\prime}$} \\
\hline & & (+) $P_{11}$ & (+) $P_{12}$ & (-) $P_{21}$ & (-) $P_{22}$ & (+) $P_{31}$ & (+) $P_{32}$ & (-) $P_{41}$ & (-) $P_{42}$ & (+) $P_{51}$ & (+) $P_{52}$ & $P_{k 1}$ & $P_{k 2}$ \\
\hline \multirow{2}{*}{$Y_{4}$} & \multirow{2}{*}{$\pi_{04}^{\prime}(1)$} & $\overline{\pi_{14}^{\prime}(-)}$ & $=0.75$ & $\pi_{24}^{\prime}(+$ & $=0.38$ & $\pi_{34}^{\prime}(+)$ & 0.00063 & $\pi_{44}^{\prime}(-$ & $=5.01$ & $\pi_{54}^{\prime}(-)$ & $=0.01$ & & \\
\hline & & $(-) P_{11}$ & $(-) P_{12}$ & $(+) P_{21}$ & $(+) P_{22}$ & $(+) P_{31}$ & $(+) P_{32}$ & $(-) P_{41}$ & $(-) P_{42}$ & $(-) P_{51}$ & $(-) P_{52}$ & $P_{k 1}$ & $P_{k 2}$ \\
\hline & & $\pi_{15}^{\prime}(+$ & $=0.46$ & $\pi_{25}^{\prime}$ & $=0.5$ & $\pi_{35}^{\prime}(-)=$ & 0.00034 & $\pi_{45}^{\prime}(+$ & $=5.57$ & $\pi_{55}^{\prime}(-)$ & $=0.01$ & & \\
\hline$Y_{5}$ & $\pi_{0}^{\prime}$ & (+) $P_{11}$ & (+) $P_{12}$ & (-) $P_{21}$ & $(-) P_{22}$ & $(-) P_{31}$ & $(-) P_{32}$ & (+) $P_{41}$ & (+) $P_{42}$ & $(-) P_{51}$ & $(-) P_{52}$ & $P_{k 1}$ & $P_{k 2}$ \\
\hline$Y$ & $\sigma^{\prime}(1)$ & $\pi_{16}^{\prime}(-)$ & 0.75 & $\pi_{26}^{\prime}(+$ & $=0.38$ & $\pi_{36}^{\prime}(-)=$ & 0.00034 & $\pi_{46}^{\prime}(+$ & $=5.57$ & $\pi_{56}^{\prime}(+$ & $=0.13$ & & \\
\hline$I_{6}$ & $\pi_{06}^{\prime}$ & $(-) P_{11}$ & $(-) P_{12}$ & $(+) P_{21}$ & $(+) P_{22}$ & $(-) P_{31}$ & $(-) P_{32}$ & $(+) P_{41}$ & $(+) P_{42}$ & $(+) P_{51}$ & $(+) P_{52}$ & $P_{k 1}$ & $P_{k 2}$ \\
\hline$\cdots$ & & & & & & & & & & & & & \\
\hline$Y$ & $\pi^{\prime}(+)$ & & & & & & & & & & & & \\
\hline$I_{n}$ & $\pi_{0 n}^{0}(+)$ & () $P_{11}$ & () $P_{12}$ & () $P_{21}$ & () $P_{22}$ & () $P_{31}$ & () $P_{32}$ & () $P_{41}$ & () $P_{42}$ & () $P_{51}$ & () $P_{52}$ & & \\
\hline
\end{tabular}

Levels of variation of the parameters of the device with a power of $7 \mathrm{~W}$ with real physical dimensions:

$$
\begin{gathered}
(-) P_{1}=6 ;(+) P_{1}=7 ;(-) P_{2}=8 ;(+) P_{2}=15 ;(-) P_{3}=4 ;(+) P_{3}=10 \\
(-) P_{4}=8 ;(+) P_{4}=15 ;(-) P_{5}=0.4 ;(+) P_{5}=0.6 ;(-) P_{6}=0.01 ;(+) P_{6}=0.05 ; \\
(-) P_{7}=40 ;(+) P_{7}=45 ;(-) P_{8}=22 ;(+) P_{8}=28 ;(-) P_{9}=60 ;(+) P_{9}=80 \\
(-) P_{10}=180 ;(+) P_{10}=220 .
\end{gathered}
$$

For a system of normal equations in a matrix form, we will write down some matrices and the relationships between them:

1) matrix of factor characteristics $\left(\pi^{\prime}\right)$ : 


$$
\pi^{\prime}=\left[\begin{array}{cccccc}
1 & 0.75 & 0.5 & 0.00034 & 5.01 & 0.011 \\
1 & 0.46 & 0.38 & 0.00034 & 5.01 & 0.131 \\
1 & 0.46 & 0.5 & 0.00063 & 5.01 & 0.131 \\
1 & 0.75 & 0.38 & 0.00063 & 5.01 & 0.011 \\
1 & 0.46 & 0.5 & 0.00034 & 5.57 & 0.011 \\
1 & 0.75 & 0.38 & 0.00034 & 5.57 & 0.131
\end{array}\right]
$$

2) transposed matrix of factor characteristics:

$$
\pi^{\prime T}=\left[\begin{array}{cccccc}
1 & 1 & 1 & 1 & 1 & 1 \\
0.75 & 0.46 & 0.46 & 0.75 & 0.46 & 0.75 \\
0.5 & 0.38 & 0.5 & 0.38 & 0.5 & 0.38 \\
3.4 \cdot 10^{-4} & 3.4 \cdot 10^{-4} & 6.3 \cdot 10^{-4} & 6.3 \cdot 10^{-4} & 3.4 \cdot 10^{-4} & 3.4 \cdot 10^{-4} \\
5.01 & 5.01 & 5.01 & 5.01 & 5.57 & 5.57 \\
0.01 & 0.13 & 0.13 & 0.01 & 0.01 & 0.13
\end{array}\right]
$$

3) on multiplying the matrices $\pi^{\prime}$ and $\pi^{\prime T}$, we obtain:

$$
\pi^{\prime} \cdot \pi^{\prime T}=\left[\begin{array}{cccccc}
6 & 3.63 & 2.64 & 2.62 \cdot 10^{-3} & 31.18 & 0.42 \\
3.63 & 2.322 & 1.58 & 1.585 \cdot 10^{-3} & 18.864 & 0.237 \\
2.64 & 1.58 & 1.183 & 1.153 \cdot 10^{-3} & 13.719 & 0.178 \\
2.62 \cdot 10^{-3} & 1.585 \cdot 10^{-3} & 1.153 \cdot 10^{-3} & 1.256 \cdot 10^{-6} & 0.014 & 1.834 \cdot 10^{-4} \\
31.18 & 18.864 & 13.719 & 0.014 & 162.45 & 2.183 \\
0.42 & 0.237 & 0.178 & 1.834 \cdot 10^{-4} & 2.183 & 0.051
\end{array}\right] \text {; }
$$

4) let us find the inverse matrix $\left(\pi^{\prime T} * \pi^{\prime}\right)^{-1}$ (correlative) [2]:

$$
=\left[\begin{array}{cccccc}
131.663 & -14.521 & -41.679 & -2.119 \cdot 10^{4} & -17.915 & 0.42 \\
-14.512 & 11.891 & 14.368 & -1.942 \cdot 10^{-10} & -1.03 \cdot 10^{-13} & 14.368 \\
-41.679 & 14.368 & 69.444 & -5.848 \cdot 10^{-10} & -2.91 \cdot 10^{-13} & 34.722 \\
-2.12 \cdot 10^{4} & -1.94 \cdot 10^{-10} & -5.85 \cdot 10^{-10} & 1.189 \cdot 10^{7} & 3.079 \cdot 10^{3} & -3.42 \cdot 10^{-10} \\
-17.915 & -1.03 \cdot 10^{-13} & -2.91 \cdot 10^{-13} & 3.079 \cdot 10^{3} & 3.189 & -1.68 \cdot 10^{-13} \\
-28.831 & 14.368 & 34.722 & -3.415 \cdot 10^{-10} & -1.68 \cdot 10^{-13} & 69.444
\end{array}\right] .
$$

Let us write down the column of parameters

$$
A=\left[\begin{array}{c}
1 \\
0.605 \\
0.44 \\
0.000485 \\
5.29 \\
0.07
\end{array}\right]
$$

5) we multiply the matrix $\pi^{\prime T} * \pi^{\prime}$ by the matrix-column of A parameters and obtain

$$
\pi^{\prime T} \cdot \pi^{\prime} \cdot A=\left[\begin{array}{c}
174.329 \\
105.537 \\
76.703 \\
0.076 \\
908.143 \\
12.191
\end{array}\right]
$$

6) let us write down the matrix-column of the outcome: 


$$
V=\left[\begin{array}{l}
0.76 \\
0.79 \\
0.68 \\
0.77 \\
0.67 \\
0.75
\end{array}\right]
$$

7) let us multiply the transposed matrix $\pi^{\prime T}$ by the matrix-column of the outcome

$$
\pi^{\prime T} V=\left[\begin{array}{c}
4.42 \\
2.694 \\
1.933 \\
1.923 \cdot 10^{-3} \\
22.939 \\
0.311
\end{array}\right] \text {; }
$$

the system of normal equations in the matrix form is written like this

$$
\pi^{\prime T} \cdot \pi^{\prime} A=\pi^{\prime T} V
$$

8) let us find the matrix-column of parameters by means of reverse matrix

$$
A=\left(\pi^{\prime T} \pi^{\prime}\right)^{-1} \pi^{\prime T} V=\left[\begin{array}{c}
1.586 \\
0.086 \\
-0.5 \\
-172.414 \\
-0.116 \\
-0.042
\end{array}\right] ;
$$

9) for all the transformations made and actions with matrices, we should bear in mind that matrix $\pi^{\prime T} \pi^{\prime}$ must be nondegenerate, and this is possible, if $\pi_{1}^{\prime}, \pi_{2}^{\prime}, \pi_{3}^{\prime}, \ldots, \pi_{k}^{\prime}$ are linearly independent.

10) if factor signs at the pair correlation give a high correlation coefficient, one of them is excluded from consideration.

In accordance with the planning matrix the experiments (calculations) are carried out and criterial regression equation is defined in the form:

$$
\xi_{v p}=1.586+0.086 \pi_{1}^{\prime}-0.5 \pi_{2}^{\prime}-172.414 \pi_{3}^{\prime}-0.116 \pi_{4}^{\prime}-0.042 \pi_{5}^{\prime} .
$$

The conducted statistical analysis of the results of the experiment revealed the homogeneity of the dispersion of experiments, the dispersion of the reproducibility of the experiment $[9 ; 10]$. Testing of the hypothesis on the adequacy of the model: $R S=3.52$ characterizes the normal distribution, as $3.12<3.52<4.12$.

The average relative error of approximation is: $\bar{e}_{\text {error }}=2.93 \%$

The obtained value of the average relative error indicates a sufficiently high level of accuracy of the constructed model, the error of less than $5 \%$ indicates a satisfactory level of accuracy.

According to the results of testing of the model on the adequacy and accuracy, the decision on the possibility of its practical use is taken.

\section{Results and discussion}

The use of various empirical coefficients and conditional indicators for the calculation and comparison of magnetic and acoustic devices depends on the individual characteristics of the experimental conditions and size of the devices. Therefore, the area of application of the above mentioned coefficients and indicators is always limited to a narrow range of structures of devices operating under certain conditions. Significant advantages over different indicators have similarity criteria. Being dimensionless complexes, they do not depend on the size of the compared structures. On the basis of the second and third similarity theorems, the number and composition of the criteria 
necessary to create new devices from those developed earlier was established. Figure 1 shows an acoustic and magnetic device installed in a geothermal boiler house, on the basis of which a model was constructed and similarity coefficients were calculated.

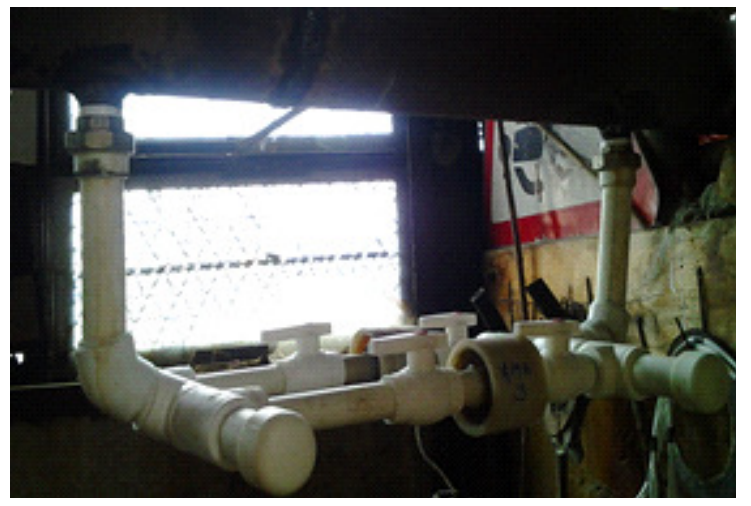

Fig.1. Exterior of devices installed on pipes in boiler room

The criteria of geometric similarity allow to calculate acoustic-magnetic devices for another geothermal well (Figure 2), and use the acoustic-magnetic device alone.

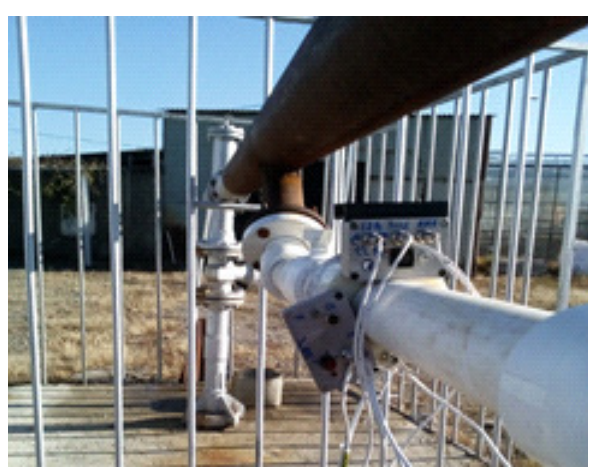

Fig. 2. Exterior of tube supplying geothermal water and device with electronic components installed on it

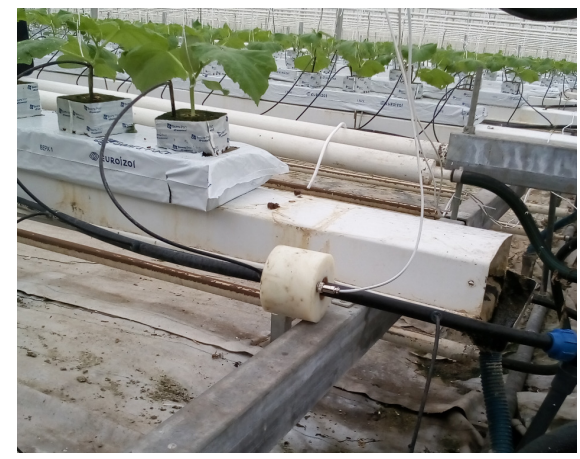

Fig. 3. Acoustic-magnetic device installed in hydroponic solution supply system

By changing the inner acoustic-magnetic device diameter and using the constructed model and similarity coefficients, it became possible to reduce the consumption of non-ferrous metals, ferromagnetic materials, body materials and the time of creating a device with significantly lower power in comparison with the prototype. Figure 3 shows the acoustic-magnetic device installed in the hydroponic solution supply system. This device was calculated on the basis of the proposed model, only the effective feature (hydrogen index) and the calculated similarity coefficients were changed.

The studies confirmed the maximum effectiveness of the feasibility of combining the methods of the theory of similarity and modeling with the methodology of planning the experiments for the design of the acoustic magnetic device [1-4].

The obtained experimental data closely coincide with the constructed model. The industrial samples of the magnetic acoustic device of the power of $100 \mathrm{~W}$ and $192 \mathrm{~W}$ installed in geothermal heating systems were calculated using the obtained coefficients of similarity.

\section{Conclusions}

The article is devoted to the investigation, which resulted in the development of the technique for studying the process of acoustic magnetic effect on the process of scale formation on the basis of combination of experimental statistical methods with the theory of similarity. To create acoustic magnetic devices of a new design, geometrical and thermal similarity were considered, similarity coefficients were established: $\pi_{1}^{\prime}(0)=0.605 ; \quad \pi_{2}^{\prime}(0)=0.44 ; \quad \pi_{3}^{\prime}(0)=0.00048 ; \quad \pi_{4}^{\prime}(0)=5.29$; $\pi_{5}^{\prime}(0)=0.07$, the choice of factors was made, the linear plan was made up, the linear model was constructed and checked for adequacy and accuracy. 
Experimental studies of a large number of devices revealed that for water with a carbonate hardness of about $7 \mathrm{mg}-\mathrm{EQ} \cdot \mathrm{l}^{-1}$, the effect of preventing scale formation in small-scale power plants is obtained with a magnetic field strength of $9.6 \cdot 10^{4}-12 \cdot 10^{4} \mathrm{~A} \cdot \mathrm{m}^{-1}$ and the water speed of $1.5-2.5 \mathrm{~m} \cdot \mathrm{s}^{-1}$. For the devices created by the model, the intensity of the magnetic field, due to the combination of acoustic and magnetic fields in the working gap, was reduced several times. Accordingly, the total power consumed by the device has decreased more than twice.

The use of the constructed model allowed to shorten the time for creation of the devices of the required capacity and eliminated the need of constructing intermediate models to refine the individual parameters. Let us note that the use of an exclusively computer model could lead to erroneous parameter values and it does not guarantee high accuracy of the model forecast.

As a result of the statistical analysis, homogeneity of the dispersion of the experiments was verified and the dispersion of the reproducibility of the experiment was determined.

\section{References}

[1] Korzhakov A.V., Korzhakov V.E., Kirillov N.P. Device for reagentless water treatment // Author's certificates for inventions BI No. 13, 1992. Author's certificate No. 1724594.

[2] Korzhakov V.E., Kramarenko B.D., Pleshakov V.V., Korzhakov A.V. Device for the non-reagent treatment of liquids // Author's certificates for inventions BI No. 38, 1989. Author's certificate No. 1514726.

[3] Korzhakov A.V., Korzhakov V.E., Oskin S.V. A device for protection against the formation of deposits on the surfaces of pipelines of the heat supply systems // Patent of the Russian Federation No. 2635591, C23F 15/00 49/06 / Application 2017100470. 09.01.2017. Publ. on 11/14/2017. Bul. No. 32.

[4] Korzhakov A.V., Korzhakov V.E., Oskin S.V. A device for protection against the formation of deposits on the surfaces of pipelines of the heat supply systems // Patent of the Russian Federation No. 2641137, C02F 1/36; C02F 1/484 Application 2017100210. 09.01.2017. Publ. on 16/01/2018. Bul. No.2.

[5] Venikov V.A. Theory of similarity and modeling (in relation to the tasks of the electric power industry): a manual for higher schools. 2nd ed., ext. and rev. - M.: Vysshaya shkola, 1976. 479 p.

[6] Kotenyov S.V. Calculation and optimization of the toroidal transformer / Kotenyov S.V., Evseev A.N. // - M.: Hot line - Telecom, 2011. 287 p.

[7] Korzhakov A.V. The development of the methods of selecting parameters of models of similar devices of acoustic and magnetic treatment of liquid/ Korzhakova S.A.: Science and Education [Text] : materials of the XVI international research and practice conference, Munich, June 27th28th, 2017 / publishing office Vela Verlag Waldkraiburg - Munich - Germany, 2017. - 132 p.R. Nicole, "Title of paper with only first word capitalized," J. Name Stand. Abbrev., in press.

[8] Korzhakov A.V. Determination of similarity criteria for the creation of new Constructions of acoustic magnetic devices designed for use in hydroponic systems. / Korzhakova S.A. // Science and Education. Materials of the XI International research and practice conference. Vol.I.April 6-7, 2016. Munich, Germany, 2016.

[9] Asaturyan V.I. Theory of experiment planning: a manual. - M.: Radio and Communication, 1983. $248 \mathrm{p}$.

[10] Spirin N.A. Planning methods and processing results of the engineering experiment: the abstract of lectures / N.A.Spirin, V. V. Lavrov. Under the General editorship of N.A. Spirina. Yekaterinburg: GOU VPO UGTU-UPI, 2004. 257 p. 